University of Wollongong

Research Online

Australian Institute for Innovative Materials -

Papers

Australian Institute for Innovative Materials

$1-1-2016$

Polarized Raman backscattering selection rules for (hhl)-oriented diamondand zincblende-type crystals

Julian Steele

University of Wollongong, js598@uowmail.edu.au

P Puech

Universite Paul Sabatier

Roger A. Lewis

University of Wollongong, roger@uow.edu.au

Follow this and additional works at: https://ro.uow.edu.au/aiimpapers

Part of the Engineering Commons, and the Physical Sciences and Mathematics Commons

Research Online is the open access institutional repository for the University of Wollongong. For further information contact the UOW Library: research-pubs@uow.edu.au 


\title{
Polarized Raman backscattering selection rules for (hhl)-oriented diamond- and zincblende-type crystals
}

\author{
Abstract \\ Due to their interesting orientation-dependent properties, the ability to grow high-index semiconductor \\ crystals and nanostructures extends the design palette for applications based on these materials. \\ Notably, a source containing a systematic reporting of what the Raman tensors are for an arbitrary high- \\ index zincblende material is yet to appear in the literature. Herein, we present the polarized Raman \\ backscattering selection rules for arbitrary (hhl)-oriented diamond- and zincblende-type crystal surfaces \\ and verify their correctness through experiment (up to (115)). Considering the many degrees of freedom \\ available to common polarized micro-Raman scattering instruments, and the unique local orientation of \\ the probed material, we further examine a range of consequences imposed by the selection rules for the \\ Raman backscattering method.

\section{Disciplines} \\ Engineering | Physical Sciences and Mathematics

\section{Publication Details} \\ Steele, J. A., Puech, P. \& Lewis, R. A. (2016). Polarized Raman backscattering selection rules for (hhl)- \\ oriented diamond- and zincblende-type crystals. Journal of Applied Physics, 120 (5), 055701-1-055701-6.
}




\title{
Polarized Raman backscattering selection rules for $(h h)$-oriented diamond- and zincblende-type crystals
}

\author{
J. A. Steele, ${ }^{1,2, a)}$ P. Puech, ${ }^{3}$ and R. A. Lewis ${ }^{1}$ \\ ${ }_{1}^{1}$ Institute for Superconducting and Electronic Materials and School of Physics, University of Wollongong, \\ Wollongong, New South Wales 2522, Australia \\ ${ }^{2}$ Centre for Surface Chemistry and Catalysis, KU Leuven, Celestijnenlaan 200F, 3001 Leuven, Belgium \\ ${ }^{3}$ CEMES-UPR8011, Université Paul Sabatier, 29 rue Jeanne Marvig, 31055 Toulouse, France
}

(Received 10 May 2016; accepted 14 July 2016; published online 1 August 2016)

\begin{abstract}
Due to their interesting orientation-dependent properties, the ability to grow high-index semiconductor crystals and nanostructures extends the design palette for applications based on these materials. Notably, a source containing a systematic reporting of what the Raman tensors are for an arbitrary high-index zincblende material is yet to appear in the literature. Herein, we present the polarized Raman backscattering selection rules for arbitrary $(h h l)$-oriented diamond- and zincblende-type crystal surfaces and verify their correctness through experiment (up to (115)). Considering the many degrees of freedom available to common polarized micro-Raman scattering instruments, and the unique local orientation of the probed material, we further examine a range of consequences imposed by the selection rules for the Raman backscattering method. Published by AIP Publishing.

[http://dx.doi.org/10.1063/1.4959824]
\end{abstract}

\section{INTRODUCTION}

Recent developments in semiconductor growth technologies have seen new innovative structures grown on ever higher crystal faces, by exploiting orientation-dependent growth procedures. ${ }^{1-3}$ The fascinating properties and useful advantages of high-index semiconductor fabrication, as well as heteroepitaxial growth atop high-index substrates, have seen growth directions as high as [119] utilized. ${ }^{2-4}$ This is largely due to the surface step structures exhibited by highindex crystal planes, which play an important role in the vapor-solid reaction processes and surface reconstruction. Certain surface reconstructions have been shown to affect the important mechanisms of epitaxial deposition ${ }^{5}$ as well as the final bulk properties. ${ }^{3}$ Studies of $(h h l)$ crystal growths misoriented between (001) and (110) planes (see Fig. 1(a) to clearly visualize such an orientation) have resulted in dramatic changes to dopant incorporation, ${ }^{4}$ while also offering altered piezoelectric properties. ${ }^{6}$ In particular, for polar systems, high-index (11 $l$ ) faces for $l>3$ have been shown to have a significantly reduced polarity; these kinds of surfaces are sometimes termed "semipolar."1

Polarized micro-Raman scattering studies have proven vital to understanding an array of emerging crystal growth techniques at the nano- and micron-scale, while being nondestructive, easy to implement and increasingly accessible to materials research scientists. ${ }^{7}$ III-V alloys often adopt the zincblende structure (point group $T_{d}$ ), which is analogous to the diamond structure (point group $O_{h}$ ), but where each atom has nearest neighbours of an unlike element. Thus, the symmetry conclusions drawn for $(h h l)$-oriented zincblende crystals will have direct implications to diamond structured materials, such as silicon. For polar zincblende alloys, the transverse (TO) and the longitudinal (LO) optical vibration

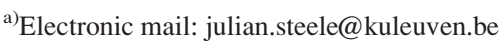

energies are different. This offers an enhancement of the information content for a given polarized Raman spectrum, due to the existence of TO and LO phonons with different frequencies, intensities, and polarization behaviour. Considering the degrees of freedom between the orientation the crystal under investigation, and the possibility of controlling polarizing optics before and after surface scattering, a unique set of Raman tensors is required to accurately explain and evaluate polarized Raman scattering experiments. For backscattering along the crystal growth axis ( $c$ axis) and the polarization vector of incident and scattered light represented, respectively, by $\hat{e}_{i}$ and $\hat{e}_{s}$, the experimental configuration is compactly described using Porto notation: ${ }^{8} \bar{c}\left(\hat{e}_{i}, \hat{e}_{s}\right) c$.

Surprisingly, the Raman scattering tensors for diamondor zincblende-type crystal indices higher than (113) are yet to be reported. In fact, this anomalous gap in scientific reporting is further worsened for the case of common non[001] growth directions, such as [111], where inconsistencies
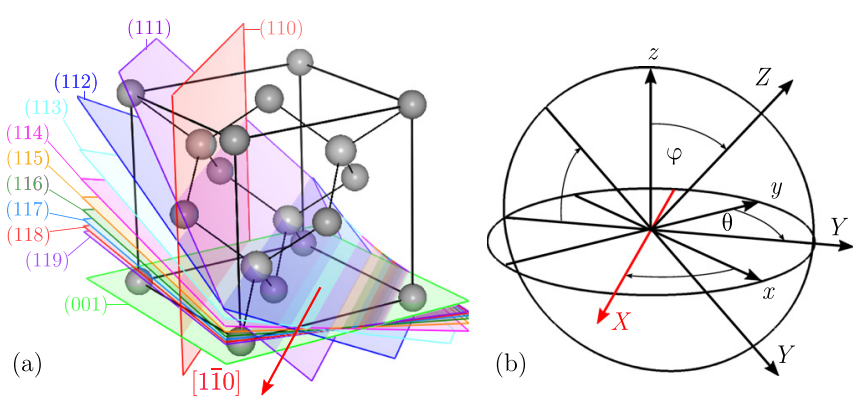

FIG. 1. (a) Zincblende/diamond structure unit cell showing the various $(h h l)$-oriented crystal planes ranging between (001) and (110), and (b) schematic representation of coordinate system used for a spatial transform between the various $c$-axes. The red $X$ axis shown in (b) and the red [1 10$]$ direction in (a) align, where crystallographic rotations around $X$ see its [1 10 ] direction maintained. The two diagrams are related through a rotation of $\varphi$ around $[1 \overline{1} 0](X)$, which translates the $Z$ through each of the $(h h l)$ planes, as depicted in (a). 
are found between different reports on what the correct Raman tensors actually are-even when employing the same coordinate basis sets. ${ }^{9,10}$ The polarization of the phonon, $Q_{j}$, should be considered in the derivation of the tensors. For the LO mode, the phonon polarization is defined as the difference between the incident photon wave-vector and the scattered light. ${ }^{11}$ The two TO mode polarizations are orthogonal to one another, with both being orthogonal to the polarization of the LO mode. With strain and lowered symmetries, it becomes more complicated and a careful analysis is required. ${ }^{12}$

In this paper, we derive the Raman scattering tensors for high-index zincblende crystals up to (119) considering phonon polarization and examine them within the context of their experimental consequences for common polarized micro-Raman backscattering configurations. Through careful measurement and analysis, polarized Raman scattering data will experimentally validate the derivation of the Raman tensors up to (115)-GaAs.

\section{RAMAN TENSORS FOR AN ARBITRARY (hh)-ORIENTED ZINCBLENDE CRYSTAL}

The nonresonant intensity of the Raman backscattering signal $I\left(\hat{e}_{i}, \hat{e}_{s}\right)$ can be related to the Raman tensor

$$
\tilde{\mathcal{R}}_{j}=\left[\begin{array}{lll}
\mathcal{R}_{x x} & \mathcal{R}_{x y} & \mathcal{R}_{x z} \\
\mathcal{R}_{y x} & \mathcal{R}_{y y} & \mathcal{R}_{y z} \\
\mathcal{R}_{z x} & \mathcal{R}_{z y} & \mathcal{R}_{z z}
\end{array}\right],
$$

which is dependent on the crystal symmetry

$$
I\left(\hat{e}_{i}, \hat{e}_{s}\right)=I_{0} \cdot \sum_{j=1}^{3}\left|\hat{e}_{i} \cdot \tilde{\mathcal{R}}_{j} \cdot \hat{e}_{s}\right|^{2} .
$$

Here, $\hat{e}_{i}$ and $\hat{e}_{s}$ represent the polarization vectors of the incident and scattered light, respectively, and $I_{0}$ contains all fixed experimental parameters (laser intensity, excitation wavelength, integration time, etc.). The index $j$ discriminates the three phonon polarization directions $x, y$, and $z$. LO phonons will vibrate in the $z$ direction, while TO phonons will vibrate along the $x$ and $y$ directions.

For a zincblende crystal possessing $T_{d}$ symmetry, we adopt the notation commonly given for the $3 \times 3$ Raman tensors in the main crystal axis system $(x=[100] ; y=[010]$; $z=[001])$, with off-diagonal elements

$$
\begin{aligned}
& \tilde{\mathcal{R}}_{x}=\left[\begin{array}{lll}
0 & 0 & 0 \\
0 & 0 & d \\
0 & d & 0
\end{array}\right] ; \quad \tilde{\mathcal{R}}_{y}=\left[\begin{array}{lll}
0 & 0 & d \\
0 & 0 & 0 \\
d & 0 & 0
\end{array}\right] ; \\
& \tilde{\mathcal{R}}_{z}=\left[\begin{array}{lll}
0 & d & 0 \\
d & 0 & 0 \\
0 & 0 & 0
\end{array}\right] .
\end{aligned}
$$

Here, $x, y$, and $z$ provide the directions of the phononinduced lattice deformation, $d$ indicates the non-zero Raman polarizability elements and are different for the LO and TO modes, i.e., $d_{\mathrm{LO}} \neq d_{\mathrm{TO}}$. For light Raman backscattered along the $z$ axis, the intensity of the LO phonon mode will be

$$
I_{\mathrm{LO}} \propto\left|\hat{e}_{i} \cdot \tilde{\mathcal{R}}_{\mathrm{LO}_{z}} \cdot \hat{e}_{s}\right|^{2},
$$

while for the case of TO phonons, the intensity is described by a proportionality to

$$
I_{\mathrm{TO}} \propto\left|\hat{e}_{i} \cdot \tilde{\mathcal{R}}_{\mathrm{TO}_{x}} \cdot \hat{e}_{s}\right|^{2}+\left|\hat{e}_{i} \cdot \tilde{\mathcal{R}}_{\mathrm{TO}_{y}} \cdot \hat{e}_{s}\right|^{2} .
$$

For an arbitrary direction, these expressions should be modified considering the phonon polarization. For example, the LO phonon polarization $\hat{e}_{\mathrm{LO}}$ is along the photon wavevector change and the Raman intensity is calculated by

$$
I_{\mathrm{LO}} \propto\left|\hat{e}_{i} \cdot\left[\tilde{\mathcal{R}}_{x} \hat{e}_{\mathrm{LO}, x}+\tilde{\mathcal{R}}_{y} \hat{e}_{\mathrm{LO}, y}+\tilde{\mathcal{R}}_{z} \hat{e}_{\mathrm{LO}, z}\right] \cdot \hat{e}_{s}\right|^{2} .
$$

A third dimension appears as the degeneracy between the LO and the TO modes is lifted and should be taken into account. Consequently, when a rotation of the Raman tensors is considered with two rotation matrices, the Raman tensors are expressed in the new basis, but the phonon polarization is still expressed in the old basis. A third rotation allows expression of the Raman tensors with their associated polarization in a new basis, and some correlation between different symmetries is even thus possible. ${ }^{11}$

For light backscattered from different $(h h l)$ crystal planes, it is useful to assign Raman tensors with respect to a coordinate system axes $(X, Y, Z)$ which are attached to the $(h h l)$-oriented crystal under investigation, as well as specify the $Z$ direction to be parallel to the incident/scattered light. A derivation of the new Raman tensors requires the application of three transformation matrices; two rotation matrices to define the new basis sets, and a final matrix associated with the phonon polarization.

Figure 1 presents both the various $(h h l)$ planes of the zincblende crystal and the coordinate system used to rotate the crystal geometry in 3D space. Given we investigate here the specific case of $(h h l)$-oriented crystal growth, and by way of mathematical convenience, we adopt an easily transformative reference frame; for $\varphi$ rotations, our [1 $1 \overline{1} 0$ ] crystal direction is systematically in-plane with the surface, as demonstrated in Figs. 1(a) and 1(b), and allocated as the $X$ axis. As high-index faces are successively accessed, a larger $\varphi$ rotation is made

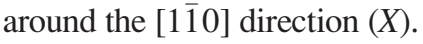

Figure 1(b) shows the unrotated laboratory coordinates $(x, y, z)$, whereby two angles of rotation, $\theta$ and $\varphi$, are made around $z$ and $X$, respectively. It follows that axis $Y$ coincides with the $[h h, 2 \bar{l}]$ direction, with the different growth orientations characterized by $\varphi=([001],[h h l])$ in the $Y$-Z plane.

To calculate the selection rules for $(h h l)$-oriented zincblende systems, appropriate Raman tensors must be transformed into the experimental coordinate system using general basis

$$
(X, Y, Z)=([1 \overline{1} 0],[l l, 2 \bar{h}],[h h l]) .
$$

The transform of the basis is made in two separate steps, whereby an initial $\theta=\pi / 4$ rotation is made around the $z=[001]$ to enter a $([1 \overline{1} 0],[110],[001])$ basis. Then, as illustrated in Fig. 1, a $\varphi$ rotation proceeds around $\mathrm{X}$ (in $\mathrm{Z}-\mathrm{Y}$ 
plane) to access the different (hhl) growth directions. These rotations can be described by the matrix

$$
A\left(\theta=\frac{\pi}{4}\right)=\left[\begin{array}{ccc}
\frac{1}{\sqrt{2}} & -\frac{1}{\sqrt{2}} & 0 \\
\frac{1}{\sqrt{2}} & \frac{1}{\sqrt{2}} & 0 \\
0 & 0 & 1
\end{array}\right]
$$

and a form of Rodrigues' rotation formula ${ }^{13}$

$$
R((\varphi))=\mathrm{I}+\sin (\varphi)[\hat{u}]_{\times}+[1-\cos (\varphi)] \hat{u} \otimes \hat{u} .
$$

Here, $A$ denotes the transformation into a system with a [110]-pointing $X$ axis, and $R$ is a rotation $\varphi$ about the $X$ axis (with unit vector $\hat{u}=\frac{1}{\sqrt{2}}[1 \overline{1} 0]$ ). Term $[\hat{u}]_{\times}$denotes the cross product matrix of $\hat{u}$ and I is the identity matrix, with

$\hat{u} \otimes \hat{u}=\left[\begin{array}{ccc}u_{x}^{2} & u_{x} u_{y} & u_{x} u_{z} \\ u_{x} u_{y} & u_{y}^{2} & u_{y} u_{z} \\ u_{x} u_{z} & u_{y} u_{z} & u_{z}^{2}\end{array}\right], \quad[\hat{u}]_{\times}=\left[\begin{array}{ccc}0 & -u_{z} & u_{y} \\ u_{z} & 0 & -u_{x} \\ -u_{y} & u_{x} & 0\end{array}\right]$.
The total transformation matrix, $T=R A$, simplifies to

$$
T=\left[\begin{array}{ccc}
\frac{\cos (\varphi)}{\sqrt{2}} & \frac{-1}{\sqrt{2}} & \frac{-\sin (\varphi)}{\sqrt{2}} \\
\frac{\cos (\varphi)}{\sqrt{2}} & \frac{1}{\sqrt{2}} & \frac{-\sin (\varphi)}{\sqrt{2}} \\
\sin (\varphi) & 0 & \cos (\varphi)
\end{array}\right],
$$

where for our cubic system

$$
\cos (\varphi)=\frac{l}{\sqrt{2 h^{2}+l^{2}}} \quad \text { and } \quad \sin (\varphi)=\frac{h \sqrt{2}}{\sqrt{2 h^{2}+l^{2}}} .
$$

The transformation matrix is orthogonal $\left(T^{T}=T^{-1}\right)$ and the transformation takes the form $\tilde{\mathcal{R}}_{j}^{\prime}=T \tilde{\mathcal{R}}_{j} T^{T}$. Multiplied by the phonon polarization in the transformed basis, $Q_{j}^{\prime}$, we arrive at the new Raman tensors for the first-order optical modes

$$
\tilde{\mathcal{R}}_{\mathrm{TO}_{X}, \mathrm{TO}_{Y}, \mathrm{LO}_{Z}}=\tilde{\mathcal{R}}_{j}^{\prime} Q_{j}^{\prime} .
$$

Here, the $j$ th term discriminates the three phonon polarization

\begin{tabular}{|c|c|c|c|c|c|c|c|c|c|c|}
\hline \multirow{2}{*}{$\begin{array}{l}\text { Crystal surface } \\
(001)\end{array}$} & \multirow{2}{*}{$\begin{array}{c}\text { Basis }(X, Y, Z) \\
\left(\left[\begin{array}{lll}1 & \overline{1} & 0\end{array},[110],[001]\right)\right.\end{array}$} & \multicolumn{9}{|c|}{ Raman tensors $\left(\mathrm{R}_{X} ; \mathrm{R}_{Y} ; \mathrm{R}_{Z}\right)$} \\
\hline & & \multicolumn{3}{|c|}{$\left[\begin{array}{ccc}0 & 0 & 1 \\
0 & 0 & 0 \\
-1 & 0 & 0\end{array}\right]$} & \multicolumn{3}{|c|}{$\left[\begin{array}{lll}0 & 0 & 0 \\
0 & 0 & 1 \\
0 & 1 & 0\end{array}\right]$} & \multicolumn{3}{|c|}{$\left[\begin{array}{ccc}-1 & 0 & 0 \\
0 & 1 & 0 \\
0 & 0 & 0\end{array}\right]$} \\
\hline (110) & $\left(\left[\begin{array}{lll}1 & \overline{1} & 0\end{array}\right],\left[\begin{array}{ll}00 & \overline{2}\end{array}\right],\left[\begin{array}{ll}110\end{array}\right]\right)$ & \multicolumn{3}{|c|}{$\left[\begin{array}{lll}0 & 1 & 0 \\
1 & 0 & 0 \\
0 & 0 & 0\end{array}\right]$} & \multicolumn{3}{|c|}{$\left[\begin{array}{ccc}1 & 0 & 0 \\
0 & 0 & 0 \\
0 & 0 & -1\end{array}\right]$} & \multicolumn{3}{|c|}{$\left[\begin{array}{ccc}0 & 0 & 0 \\
0 & 0 & -1 \\
0 & -1 & 0\end{array}\right]$} \\
\hline (111) & $\left(\left[\begin{array}{lll}1 & \overline{1} & 0],[11 \overline{2}],[111])\end{array}\right.\right.$ & {$\left[\begin{array}{c}0 \\
0.8165 \\
-0.5774\end{array}\right.$} & $\begin{array}{c}0.8165 \\
0 \\
0\end{array}$ & $\left.\begin{array}{c}-0.5774 \\
0 \\
0\end{array}\right]$ & {$\left[\begin{array}{c}0.8165 \\
0 \\
0\end{array}\right.$} & $\begin{array}{c}0 \\
-0.8165 \\
-0.5774\end{array}$ & $\left.\begin{array}{c}0 \\
-0.5774 \\
0\end{array}\right]$ & {$\left[\begin{array}{c}-0.5774 \\
0 \\
0\end{array}\right.$} & $\begin{array}{c}0 \\
-0.5774 \\
0\end{array}$ & $\begin{array}{c}0 \\
0 \\
1.1547\end{array}$ \\
\hline$(112)$ & $\left(\left[\begin{array}{lll}1 & \overline{1} & 0\end{array}\right],\left[\begin{array}{ll}22 & \overline{2}\end{array}\right],\left[\begin{array}{ll}112\end{array}\right]\right)$ & {$\left[\begin{array}{c}0 \\
0.5774 \\
-0.8165\end{array}\right.$} & $\begin{array}{c}0.5774 \\
0 \\
0\end{array}$ & $\left.\begin{array}{c}-0.8165 \\
0 \\
0\end{array}\right]$ & {$\left[\begin{array}{c}0.5774 \\
0 \\
0\end{array}\right.$} & $\begin{array}{c}0 \\
-1.1547 \\
0\end{array}$ & $\left.\begin{array}{c}0 \\
0 \\
0.5774\end{array}\right]$ & {$\left[\begin{array}{c}-0.8165 \\
0 \\
0\end{array}\right.$} & $\begin{array}{c}0 \\
0 \\
0.5774\end{array}$ & $\left.\begin{array}{c}0 \\
0.5774 \\
0.8165\end{array}\right]$ \\
\hline (113) & $\left(\left[\begin{array}{lll}1 & \overline{1} & 0\end{array}\right],\left[\begin{array}{ll}33 & 2\end{array}\right],[113]\right)$ & {$\left[\begin{array}{c}0 \\
0.4264 \\
-0.9045\end{array}\right.$} & $\begin{array}{c}0.4264 \\
0 \\
0\end{array}$ & $\left.\begin{array}{c}-0.9045 \\
0 \\
0\end{array}\right]$ & {$\left[\begin{array}{c}0.4264 \\
0 \\
0\end{array}\right.$} & $\begin{array}{c}0 \\
-1.0466 \\
0.4112\end{array}$ & $\left.\begin{array}{c}0 \\
0.4112 \\
0.6202\end{array}\right]$ & {$\left[\begin{array}{c}-0.9045 \\
0 \\
0\end{array}\right.$} & $\begin{array}{c}0 \\
0.4112 \\
0.6202\end{array}$ & $\left.\begin{array}{c}0 \\
0.6202 \\
0.4934\end{array}\right]$ \\
\hline$(114)$ & $\left(\left[\begin{array}{lll}1 & \overline{1} & 0\end{array}\right],\left[\begin{array}{ll}44 & \overline{2}\end{array}\right],[114]\right)$ & {$\left[\begin{array}{c}0 \\
0.3333 \\
-0.9428\end{array}\right.$} & $\begin{array}{c}0.333 \\
0 \\
0\end{array}$ & $\left.\begin{array}{c}-0.9428 \\
0 \\
0\end{array}\right]$ & {$\left[\begin{array}{c}0.3333 \\
0 \\
0\end{array}\right.$} & $\begin{array}{c}0 \\
-0.8889 \\
0.6285\end{array}$ & $\left.\begin{array}{c}0 \\
0.6285 \\
0.5556\end{array}\right]$ & {$\left[\begin{array}{c}-0.9428 \\
0 \\
0\end{array}\right.$} & $\begin{array}{c}0 \\
0.6285 \\
0.5556\end{array}$ & $\left.\begin{array}{c}0 \\
0.5556 \\
0.3143\end{array}\right]$ \\
\hline$(115)$ & $\left(\left[\begin{array}{lll}1 & \overline{1} & 0\end{array}\right],\left[\begin{array}{ll}55 & \overline{2}\end{array}\right],\left[\begin{array}{ll}115\end{array}\right]\right)$ & {$\left[\begin{array}{c}0 \\
0.2722 \\
-0.9623\end{array}\right.$} & $\begin{array}{c}0.2722 \\
0 \\
0\end{array}$ & $\left.\begin{array}{c}-0.9623 \\
0 \\
0\end{array}\right]$ & {$\left[\begin{array}{c}0.2722 \\
0 \\
0\end{array}\right.$} & $\begin{array}{c}0 \\
-0.7560 \\
0.7484\end{array}$ & $\left.\begin{array}{c}0 \\
0.7484 \\
0.4838\end{array}\right]$ & {$\left[\begin{array}{c}-0.9623 \\
0 \\
0\end{array}\right.$} & $\begin{array}{c}0 \\
0.7484 \\
0.4838\end{array}$ & $\left.\begin{array}{c}0 \\
0.4838 \\
0.2138\end{array}\right]$ \\
\hline$(116)$ & $\left(\left[\begin{array}{lll}1 & \overline{1} & 0\end{array}\right],\left[\begin{array}{ll}66 & \overline{2}\end{array}\right],[116]\right)$ & {$\left[\begin{array}{c}0 \\
0.2294 \\
-0.9733\end{array}\right.$} & $\begin{array}{c}0.2294 \\
0 \\
0\end{array}$ & $\left.\begin{array}{c}-0.9733 \\
0 \\
0\end{array}\right]$ & {$\left[\begin{array}{c}0.2294 \\
0 \\
0\end{array}\right.$} & $\begin{array}{c}0 \\
-0.6520 \\
0.8196\end{array}$ & $\left.\begin{array}{c}0 \\
0.8196 \\
0.4226\end{array}\right]$ & {$\left[\begin{array}{c}-0.9733 \\
0 \\
0\end{array}\right.$} & $\begin{array}{c}0 \\
0.8196 \\
0.4226\end{array}$ & $\left.\begin{array}{c}0 \\
0.4226 \\
0.1537\end{array}\right]$ \\
\hline$(117)$ & $\left(\left[\begin{array}{lll}1 & \overline{1} & 0\end{array}\right],\left[\begin{array}{ll}77 & \overline{2}\end{array}\right],[117]\right)$ & {$\left[\begin{array}{c}0 \\
0.1980 \\
-0.9802\end{array}\right.$} & $\begin{array}{c}0.1980 \\
0 \\
0\end{array}$ & $\left.\begin{array}{c}-0.9802 \\
0 \\
0\end{array}\right]$ & {$\left[\begin{array}{c}0.1980 \\
0 \\
0\end{array}\right.$} & $\begin{array}{c}0 \\
-0.5708 \\
0.8649\end{array}$ & $\left.\begin{array}{c}0 \\
0.8649 \\
0.3728\end{array}\right]$ & {$\left[\begin{array}{c}-0.9802 \\
0 \\
0\end{array}\right.$} & $\begin{array}{c}0 \\
0.8649 \\
0.3728\end{array}$ & $\left.\begin{array}{c}0 \\
0.3728 \\
0.1153\end{array}\right]$ \\
\hline (118) & $\left(\left[\begin{array}{lll}1 & \overline{1} & 0\end{array}\right],\left[\begin{array}{ll}88 & \overline{2}\end{array}\right],\left[\begin{array}{ll}118\end{array}\right)\right.$ & {$\left[\begin{array}{c}0 \\
0.1741 \\
-0.9847\end{array}\right.$} & $\begin{array}{c}0.1741 \\
0 \\
0\end{array}$ & $\left.\begin{array}{c}-0.9847 \\
0 \\
0\end{array}\right]$ & {$\left[\begin{array}{c}0.1741 \\
0 \\
0\end{array}\right.$} & $\begin{array}{c}0 \\
-0.5064 \\
0.8952\end{array}$ & $\left.\begin{array}{c}0 \\
0.8952 \\
0.3323\end{array}\right]$ & {$\left[\begin{array}{c}-0.9847 \\
0 \\
0\end{array}\right.$} & $\begin{array}{c}0 \\
0.8952 \\
0.3323\end{array}$ & $\left.\begin{array}{c}0 \\
0.3323 \\
0.0895\end{array}\right]$ \\
\hline (119) & $\left(\left[\begin{array}{lll}1 & \overline{1} & 0\end{array},\left[\begin{array}{ll}99 & \overline{2}\end{array}\right],[119]\right)\right.$ & {$\left[\begin{array}{c}0 \\
0.1552 \\
-0.9879\end{array}\right.$} & $\begin{array}{c}0.1552 \\
0 \\
0\end{array}$ & $\left.\begin{array}{c}-0.9879 \\
0 \\
0\end{array}\right]$ & {$\left[\begin{array}{c}0.1552 \\
0 \\
0\end{array}\right.$} & $\begin{array}{c}0 \\
-0.4545 \\
0.9165\end{array}$ & $\left.\begin{array}{c}0 \\
0.9165 \\
0.2992\end{array}\right]$ & {$\left[\begin{array}{c}-0.9879 \\
0 \\
0\end{array}\right.$} & $\begin{array}{c}0 \\
0.9165 \\
0.2992\end{array}$ & $\left.\begin{array}{c}0 \\
0.2992 \\
0.0714\end{array}\right]$ \\
\hline
\end{tabular}
directions $X, Y$, and $Z$. Inserting the rotated Raman tensors into Eq. (2), one can proceed to simply determine the Raman activity of LO and TO vibrations through Eqs. (4) and (5).

TABLE I. Raman tensors for $(h h l)$-oriented zincblende crystals. 
Implementing this procedure, the Raman tensors are calculated (in units of the Raman polarizability, $d_{\mathrm{LO}, \mathrm{TO}}$ ) for crystal faces up to the (119) plane and are provided in Table I.

\section{EXPERIMENTAL CONSEQUENCES OF POLARIZED RAMAN BACKSCATTERING CONFIGURATIONS}

It was shown useful in Section II to specify Raman tensors based on a coordinate system of axes (X, Y, and Z) which are attached to the investigated microstructure. However, the polarization vector terms in Eq. (2) for incident $\left(\hat{e}_{i}\right)$ and scattered light $\left(\hat{e}_{s}\right)$ are yet to receive detailed treatment. Figure 2 shows the geometry of a polarized Raman backscattering configuration and relates the employed basis coordinate system to the laboratory coordinates, with backscattering occurring in the $\pm Z$ direction $(Z\|c\| k)$. In the laboratory coordinates, the polarization of incident and scattered radiation takes the form

$$
\hat{e}_{i}=\left[\begin{array}{c}
\sin (\alpha) \\
\cos (\alpha) \\
0
\end{array}\right] \text { and } \quad \hat{e}_{s}=\left[\begin{array}{c}
\sin (\alpha+\beta) \\
\cos (\alpha+\beta) \\
0
\end{array}\right] .
$$

Here, angles $\alpha$ and $\beta$ are measured relative to $Y$, and are realized using a rotation stage and rotating polarizer (analyzer), respectively. Thus, all rotations are made relative to a fixed $\hat{e}_{i}$, which is typically to avoid problems introduced by the intrinsic polarization of the laser light source (solid-state, ion, or otherwise). It follows, for the general case, the intensity of the measured first-order optical phonon bands is described by

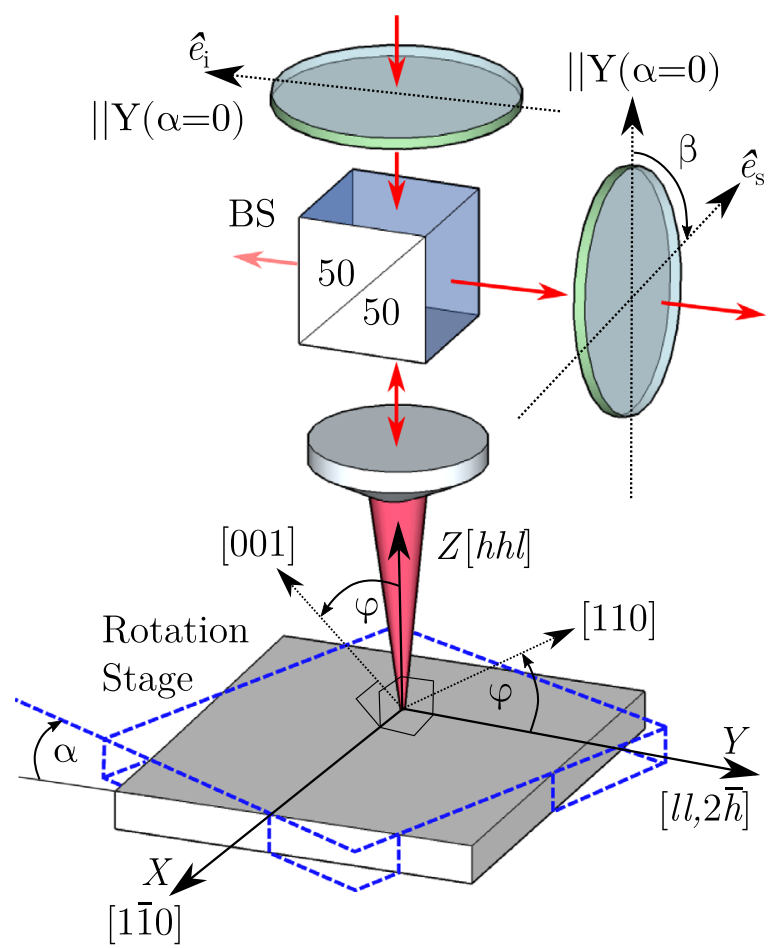

FIG. 2. Schematic diagram of typical micro-Raman experimental configuration and the coordinate systems employed in the backscattering geometry from $(h h l)$-oriented crystal faces. Here, $\hat{e}_{i}$ and $\hat{e}_{s}$ indicates the polarization of the incident and scattered light, respectively. The other terms are explained in the text.
TABLE II. Raman backscattering selection rules for different experimental configurations given in terms of generalized Raman tensor elements. Note the symmetric nature of elements $\mathcal{R}_{X Y}$ and $\mathcal{R}_{Y X}\left(\mathcal{R}_{X Y}=\mathcal{R}_{Y X}\right)$ act to simplify the rules.

\begin{tabular}{lc}
\hline \hline Polarized configuration & Selection rules $I_{\mathrm{LO}} \propto I_{\mathrm{LO}_{Z}} ; I_{\mathrm{TO}} \propto I_{\mathrm{TO}_{X}}+I_{\mathrm{TO}_{Y}}$ \\
\hline$Z(Y Y) \bar{Z}$ & $\left|\mathcal{R}_{Y Y}\right|^{2}$ \\
$Z(Y X) \bar{Z}$ & $\left|\mathcal{R}_{X Y}\right|^{2}$ \\
$Z(X X) \bar{Z}$ & $\left|\mathcal{R}_{X X}\right|^{2}$ \\
$Z(X Y) \bar{Z}$ & $\left|\mathcal{R}_{X Y}\right|^{2}$ \\
$\hat{e}_{i} \| \hat{e}_{s}(\alpha)$ & $\left|\mathcal{R}_{X X} \sin ^{2}(\alpha)+\mathcal{R}_{Y Y} \cos ^{2}(\alpha)+\mathcal{R}_{X Y} \sin (2 \alpha)\right|^{2}$ \\
$\hat{e}_{i} \perp \hat{e}_{s}(\alpha)$ & $\left|\sin (\alpha) \mathcal{R}_{X X}+\cos (\alpha) \mathcal{R}_{Y X}\right|^{2}$ \\
$\hat{e}_{i} \| Y, \hat{e}_{s}(\beta)$ & $\left|\mathcal{R}_{X Y} \sin (\beta)+\mathcal{R}_{Y Y} \cos (\beta)\right|^{2}$ \\
$\hat{e}_{i} \| X, \hat{e}_{s}(\beta)$ & $\left|\mathcal{R}_{X X} \cos (\beta)+\mathcal{R}_{X Y} \sin (\beta)\right|^{2}$ \\
\hline \hline
\end{tabular}

$I \propto\left|[\sin (\alpha) \cos (\alpha) 0]\left[\begin{array}{lll}\mathcal{R}_{X X} & \mathcal{R}_{X Y} & \mathcal{R}_{X Z} \\ \mathcal{R}_{Y X} & \mathcal{R}_{Y Y} & \mathcal{R}_{Y Z} \\ \mathcal{R}_{Z X} & \mathcal{R}_{Z Y} & \mathcal{R}_{Z Z}\end{array}\right]\left[\begin{array}{c}\sin (\alpha+\beta) \\ \cos (\alpha+\beta) \\ 0\end{array}\right]\right|^{2}$.

We assume in our case that the LO mode is along $\mathrm{Z}$. Consequently, the associated polarization of each Raman tensor is either a TO mode or a LO mode. If the numerical aperture of a microscope is considered, the photon wavevector is not always fully parallel to the $\mathrm{Z}$ axis. Thus, a small disorientation should be introduced leading to a mixing of the Raman tensors.

Equation (14) contains all the degrees of freedom possessed in a standard Raman backscattering measurement. For $\beta$ angles of 0 and $1 / \pi, \hat{e}_{i}$ and $\hat{e}_{s}$ will be parallel and perpendicular, respectively. Thus, an appropriate $\alpha$ rotation in these two geometries will allow access to the four main polarized configurations of backscattering measurements, which are presented with their respective selection rules in the first four rows of Table II. Fixing $\hat{e}_{i}$ and $\hat{e}_{s}$ to be parallel or perpendicular, and rotating the sample through $\alpha$, will yield the polartype measurements described by rows 5 and 6 , respectively. Polarizing the incident light $\hat{e}_{i}$ in either the $Y$ or $X$ directions

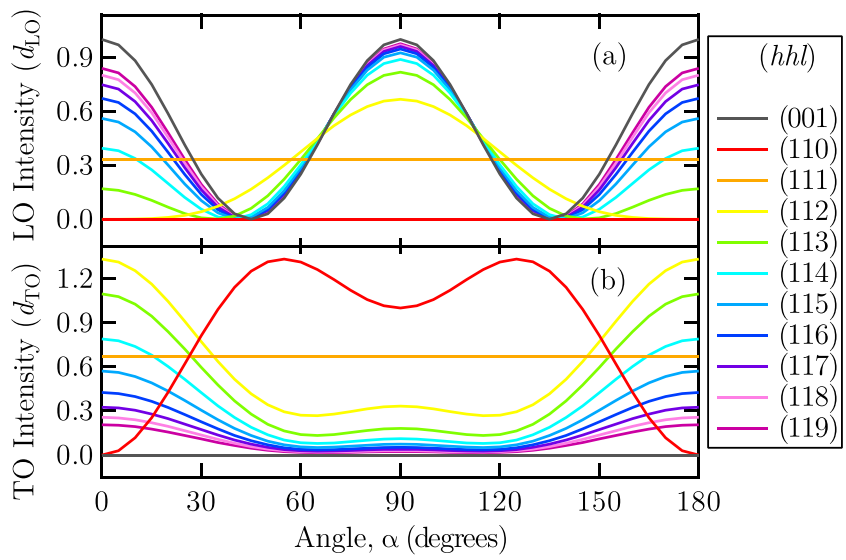

FIG. 3. Calculated first-order Raman scattering intensity from a diamond- or zincblende-type crystal using a parallel-polarized excitation and detection geometry $\left(\hat{e}_{i} \| \hat{e}_{s}\right)$ as a function of a rotation around $Z, \alpha$. (a) intensity of measured LO phonon band, and (b) intensity of the TO phonon modes with contributions from both perpendicular displacements $(X$ and $Y$ ). 
(via the necessary $\alpha$ rotation) and rotating the analyzer through $\beta$ will, respectively, generate the bottom rows, 7 and 8 .

Because the alignment of $\hat{e}_{i}$ or $\hat{e}_{s}$ with either $\mathrm{X}$ or $\mathrm{Y}$ basis direction is not assured for a system of unknown orientation, $I\left(\hat{e}_{i} \| \hat{e}_{s}(\alpha)\right)$ measurements rotated through $\alpha$ usefully abnegates the need to establish such a reference direction; this can be determined by the analysis. To investigate unknown crystal orientations, the $\hat{e}_{i} \| \hat{e}_{s}(\alpha)$ polarized Raman backscattering geometry is commonly employed by researchers.

Figure 3 displays the theoretical intensity of LO and TO modes measured in this configuration, as a function of the sample rotation, $\alpha$. Examining these data reveals several general rules for polarized Raman backscattering from different $(h h l)$ zincblende surfaces. First, for light backscattered from a (001) face, only LO phonon modes are active, with the TO band being symmetry forbidden. Conversely, the Raman signal from (110)-oriented crystals should only contain contributions from TO phonons, with the LO mode symmetry forbidden for this surface. On the other hand, for Raman backscattering in the high-symmetry [111] direction, both LO and TO modes are active, with intensities that are invariant to changes in $\alpha$. As the crystal plane rotates away from (111) to the high index (112) plane, and higher, through a larger $\varphi$ rotation, we see a transition in general scattering behavior. Here, the planes become more (001)-like when approaching the (119) plane; for $l \rightarrow \infty,(11 l) \rightarrow(001)$. As one would expect examining the relationship between the various $(h h l)$ crystal planes in Fig. 1(a), the exhibited changes in the scattering intensities of LO and TO phonons are very systematic and distinguished.
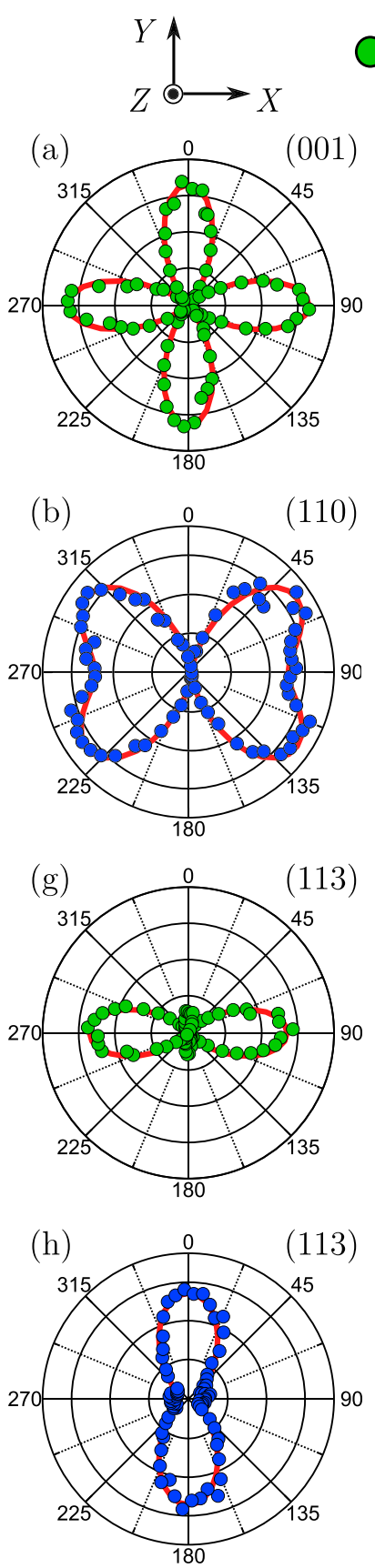

\section{LO phonon}

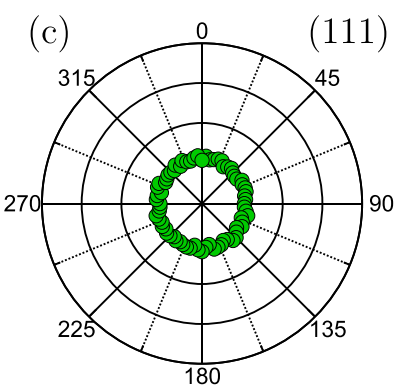

(d)
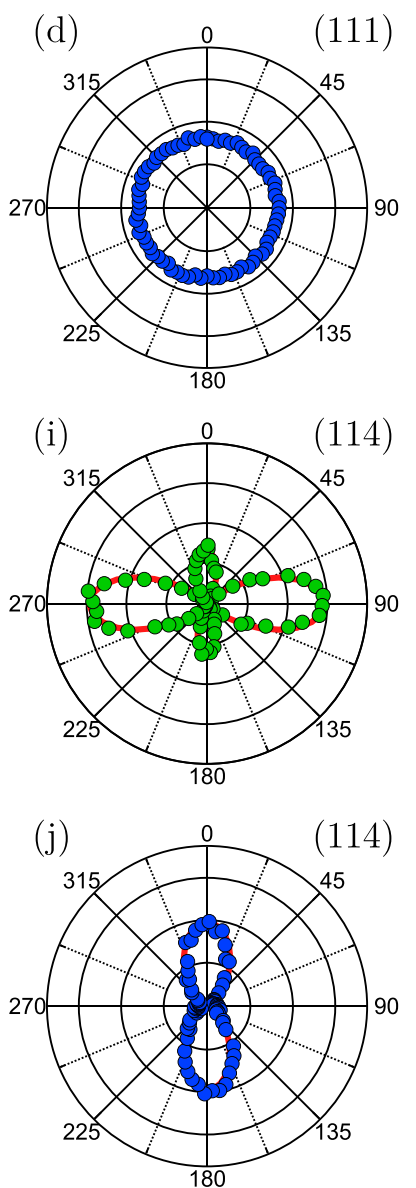
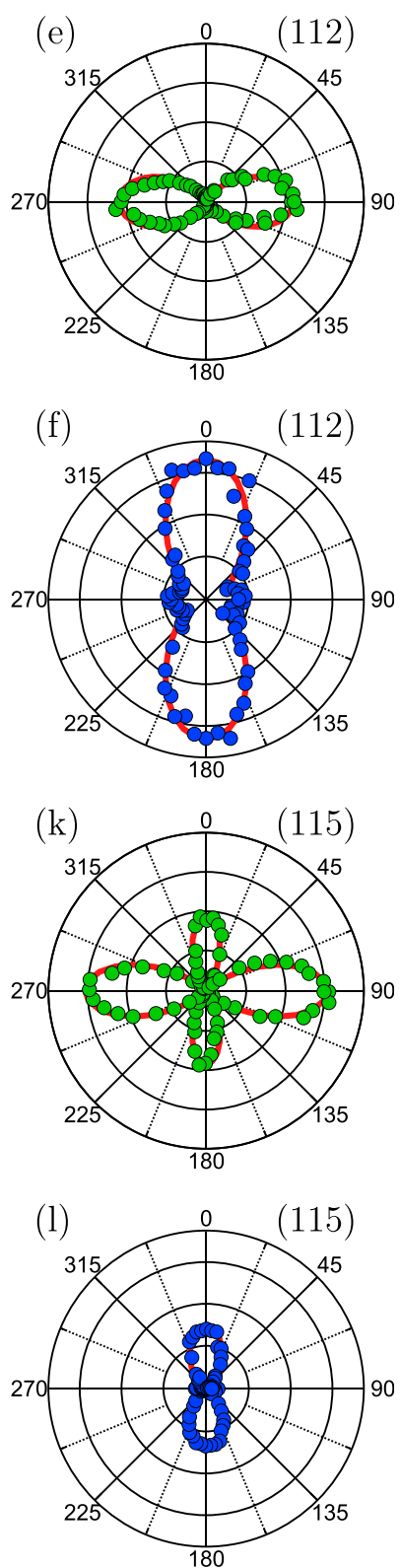

FIG. 4. Polar plots of calculated and experimental Raman data measured in a $I\left(\hat{e}_{i} \| \hat{e}_{s}(\alpha)\right)$ configuration from bulk (a) (001), (b) (110), (c) and (d) (111), (e) and (f) (112), (g) and (h) (113), (i) and (j) (114), and (k) and (l) (115) GaAs. 
Next we verify the derived Raman tensors for $(h h l)$ zincblende-type crystals by measuring the polarized Raman backscattering spectra from high-index ( $h h l)$-zincblende crystals and compare the experimental data to theoretical calculations. Our bulk undoped GaAs samples possessed the following substrate orientations: (001), (110), (111), (112), (113), (114), and (115). Polarized Raman backscattering acquisitions have been performed using a Jobin-Yvon HR800 micro-Raman system with $632.8 \mathrm{~nm}$ excitation and $\times 10$ objective. The experimental geometry implemented for these measurements is the $\hat{e}_{i} \| \hat{e}_{s}(\alpha)$ configuration, which is described by selection rules presented in Table II. Regardless of the relative direction between the polarizer, analyzer and the studied crystal, this geometry offers great utility in determining unknown crystal orientation from one $\alpha=\pi$ rotation sequence.

Polar plots of the first-order optical Raman peaks for GaAs are presented in Fig. 4 for a full $\alpha=2 \pi$ rotation, along with their calculated values. The data are described by the coordinate system (top of figure) and the generalized basis sets in Eq. (7). For completeness, note that the forbidden vibrational modes (LO and TO bands from (110) and (001) planes, respectively) are not included in these data. There is excellent agreement here between theory and experiment, confirming the methodology to derive the Raman tensors. Of the inconsistencies in the literature for tensors describing [111] Raman backscattering, it becomes clear those reported by Puech et al. ${ }^{10}$ are accurate, while Bulkin et al..$^{9}$ appears to be incorrect. Backscattering along our defined $Z$ basis (neither the $\hat{e}_{i}$ or $\hat{e}_{s}$ vector has a component in the $Z$ direction) means that the only tensor elements used for the calculation of the optical phonon intensities are $x, x, x, y, y, x$, and $y, y$. It follows that the symmetry of the tensors provided by Bulkin et $a l^{9}{ }^{9}$ for a (111)-oriented zincblende crystal provide an angular oscillation in intensity, rather than reflecting the high-symmetry character (angle independent in current geometry) of the [111] crystallographic direction. Further, Fig. 4 reiterates the systematic nature of changes seen in the LO and TO phonons for an increasing crystal index $(l \rightarrow \infty)$; the Raman selection rules for the optical modes become more (001)-like at our highest (115)-oriented GaAs crystal.

The goal of this work is to clearly verify and layout the Raman selection rules for ( $h h l$ )-oriented GaAs crystals. However, in terms of the useful characterization of polar crystal, the most significant parameters of the Raman signature are the first-order optical bands, whose relative strength $\left(I_{\mathrm{LO}} / I_{\mathrm{TO}}\right)$ depend on the crystalline state of the system. Within this context, an analysis of the ratio of the TO and LO phonon intensities for a given geometry will yield insights into lattice perfection/quality. For the current example of GaAs, it has been shown that misorientation and disorder effects may contribute strongly to this ratio. ${ }^{14}$ This can be of importance when attempting to use it as a quantitative probe for local crystal properties.

\section{CONCLUSION}

We have presented a general derivation of the polarized Raman backscattering selection rules for an arbitrary $(h h l)$ oriented zincblende-type crystal surface. A direct comparison of the theory and Raman backscattering experiments performed on high-index GaAs crystals (up to (115)) has been carefully conducted. Considering the degrees of freedom available for both the orientation of the investigated material system and the possibility to employ polarizing optics before and after the backscattering surface, we examined the full range of consequences imposed by the selection rules for common Raman backscattering configurations.

\section{ACKNOWLEDGMENTS}

The authors would like to thank Professor M. Henini from the University of Nottingham for providing the GaAs samples used in this study. J.S. acknowledges the Research Career Launch Scholarship funding provided by the University of Wollongong.

${ }^{1}$ R. Ravash, J. Blaesing, A. Dadgar, and A. Krost, "Semipolar single component GaN on planar high index $\mathrm{Si}(11 \mathrm{~h})$ substrates," Appl. Phys. Lett. 97, 142102 (2010).

${ }^{2}$ M. Schmidbauer, S. Seydmohamadi, D. Grigoriev, Z. M. Wang, Y. I. Mazur, P. Schäfer, M. Hanke, R. Köhler, and G. J. Salamo, "Controlling planar and vertical ordering in three-dimensional (In,Ga)As quantum dot lattices by GaAs surface orientation," Phys. Rev. Lett. 96, 066108 (2006). ${ }^{3}$ B. L. Liang, Z. M. Wang, Y. I. Mazur, V. V. Strelchuck, K. Holmes, J. H. Lee, and G. J. Salamo, "InGaAs quantum dots grown on B-type high index GaAs substrates: Surface morphologies and optical properties," Nanotechnology 17, 2736-2740 (2006).

${ }^{4}$ H. Beneking, P. Narozny, and N. Emeis, "High quality epitaxial GaAs and InP wafers by isoelectronic doping," Appl. Phys. Lett. 47, 828-830 (1985).

${ }^{5}$ M. Henini, J. Ibáñez, M. Schmidbauer, M. Shafi, S. V. Novikov, L. Turyanska, S. I. Molina, D. L. Sales, M. F. Chisholm, and J. Misiewicz, "Molecular beam epitaxy of GaBiAs on (311)B GaAs substrates," Appl. Phys. Lett. 91, 251909 (2007).

${ }^{6}$ A. Patanè, A. Levin, A. Polimeni, F. Schindler, P. C. Main, L. Eaves, and M. Henini, "Piezoelectric effects in $\mathrm{In}_{0.5} \mathrm{Ga}_{0.5}$ As self-assembled quantum dots grown on (311)B GaAs substrates," Appl. Phys. Lett. 77, 2979 (2000).

${ }^{7} \mathrm{G}$. Turrell and J. Corset, Raman Microscopy: Developments and Applications (Academic Press, 1996).

${ }^{8}$ T. C. Damen, S. P. S. Porto, and B. Tell, "Raman effect in zinc oxide," Phys. Rev. 142, 570-574 (1966).

${ }^{9}$ Analytical Raman Spectroscopy, edited by J. G. Bulkin and B. J. Grasselli (John Wiley, New York, 1991).

${ }^{10}$ P. Puech, G. Landa, R. Carles, P. S. Pizani, E. Daran, and C. Fontaine, "Strain relaxation in [001]- and [111]-GaAs/ $\mathrm{CaF}_{2}$ analyzed by Raman spectroscopy,” J. Appl. Phys. 77, 1126-1132 (1995).

${ }^{11}$ P. Puech, G. Landa, R. Carles, and C. Fontaine, "Strain effects on optical phonons in $\langle 111\rangle$ GaAs layers analyzed by Raman scattering," J. Appl. Phys. 82, 4493-4499 (1997).

${ }^{12}$ E. Anastassakis, "Selection rules of Raman scattering by optical phonons in strained cubic crystals,” J. Appl. Phys. 82, 1582-1591 (1997).

${ }^{13}$ O. Rodrigues, “Des lois géométriques qui régissent les déplacements d'un système solide dans l'espace, et de la variation des coordonnées provenant de ces déplacements considérés indépendamment des causes qui peuvent les produire," J. Math. Pures Appl. 8, 380-440 (1840).

${ }^{14}$ J. Biellmann, B. Prevot, and C. Schwab, "First-order Raman line intensity ratio in GaAs: A potential lattice perfection scale," J. Phys. C: Solid State Phys. 16, 1135-1142 (1983). 